\title{
Universality and the dynamical space-time dimensionality in the Lorentzian type IIB matrix model
}

\author{
Yuta Ito, ${ }^{a}$ Jun Nishimura ${ }^{a, b}$ and Asato Tsuchiya ${ }^{c}$ \\ ${ }^{a}$ KEK Theory Center, High Energy Accelerator Research Organization, \\ 1-1 Oho, Tsukuba, Ibaraki 305-0801, Japan \\ ${ }^{b}$ Graduate University for Advanced Studies (SOKENDAI), \\ 1-1 Oho, Tsukuba, Ibaraki 305-0801, Japan \\ ${ }^{c}$ Department of Physics, Shizuoka University, \\ 836 Ohya, Suruga-ku, Shizuoka 422-8529, Japan \\ E-mail: yito@post.kek.jp, jnishi@post.kek.jp, \\ tsuchiya.asato@shizuoka.ac.jp
}

Abstract: The type IIB matrix model is one of the most promising candidates for a nonperturbative formulation of superstring theory. In particular, its Lorentzian version was shown to exhibit an interesting real-time dynamics such as the spontaneous breaking of the 9-dimensional rotational symmetry to the 3-dimensional one. This result, however, was obtained after regularizing the original matrix integration by introducing "infrared" cutoffs on the quadratic moments of the Hermitian matrices. In this paper, we generalize the form of the cutoffs in such a way that it involves an arbitrary power $(2 p)$ of the matrices. By performing Monte Carlo simulation of a simplified model, we find that the results become independent of $p$ and hence universal for $p \gtrsim 1.3$. For $p$ as large as 2.0, however, we find that large- $N$ scaling behaviors do not show up, and we cannot take a sensible large- $N$ limit. Thus we find that there is a certain range of $p$ in which a universal large- $N$ limit can be taken. Within this range of $p$, the dynamical space-time dimensionality turns out to be $(3+1)$, while for $p=2.0$, where we cannot take a sensible large- $N$ limit, we observe a $(5+1)$ d structure.

KeYwORDs: Matrix Models, 1/N Expansion

ARXIV EPRINT: 1701.07783 


\section{Contents}

1 Introduction 1

2 The Lorentzian type IIB matrix model with generalized IR cutoffs 3

3 Universality in the results for various $p \quad 6$

4 Probing the IR cutoff effects by the SDE 10

$5 \quad$ Absence of large- $N$ scaling behavior for $p=2.0 \quad 12$

6 Summary 13

$\begin{array}{ll}\text { A Results for the spatial SDE } & 15\end{array}$

\section{Introduction}

Since its proposal in 1996 [1], the type IIB matrix model has been studied from various angles as a possible nonperturbative formulation of superstring theory. Formally it resembles the proposals in refs. $[2,3]$ in the sense that all the models can be obtained by dimensionally reducing $10 \mathrm{D} \mathcal{N}=1$ super Yang-Mills action to lower dimensions. From this point of view, the type IIB matrix model may be viewed as an extreme case since the dimensional reduction is conducted down to $d=0$. This makes the model distinct in that not only space but also time is treated as an emergent concept that appears from the matrix degrees of freedom, and consequently the model enjoys manifest Lorentz invariance. There is also a strong evidence that the model can reproduce the perturbation theory of type IIB superstring theory to all orders in the string coupling constant [4].

In the literature, the type IIB matrix model was studied mostly after making a "Wick rotation" $A_{0}=i A_{10}$, where $A_{0}$ represents the matrix corresponding to the time. The Euclidean version obtained in this way has a positive semi-definite action for the bosonic part, and the partition function is proved to be finite in spite of the existence of flat directions $[5,6]$. The $\mathrm{SO}(10)$ symmetry of the model is expected to be spontaneously broken down to $\mathrm{SO}(4)$ in order to realize the dynamical generation of four-dimensional space-time [7]. The latest result obtained by the Gaussian expansion method suggests, however, that it is broken down to $\mathrm{SO}(3)$, and the extent of space in the extended directions is only five times larger than the shrunken directions [8].

The Lorentzian version of the type IIB matrix model, on the other hand, is not welldefined as it is since the bosonic part of the action is not positive semi-definite. In ref. [9], the model was studied by Monte Carlo simulation after regularizing the matrix integral by introducing "infrared" cutoffs on $\operatorname{tr}\left(A_{0}\right)^{2}$ and $\sum_{i=1}^{9} \operatorname{tr}\left(A_{i}\right)^{2}$ for the temporal and spatial 
directions, respectively. The matrix configurations obtained by the simulation were found to have an approximate band-diagonal structure in the basis which diagonalizes the temporal matrix as $A_{0}=\operatorname{diag}\left(\alpha_{1}, \cdots, \alpha_{N}\right)$ with the order $\alpha_{1}<\cdots<\alpha_{N}$. Therefore it makes sense to identify the state at time $\alpha_{a}$ by cutting out a block matrix from $A_{i}(i=1, \cdots, 9)$ around the diagonal element at the position of $\alpha_{a}$ in $A_{0}$. The real-time evolution extracted in this way showed the following exciting behavior: the extent of space in three out of nine directions starts to grow at some point in time indicating that the $\mathrm{SO}(9)$ rotational symmetry of the spatial matrices is broken down to $\mathrm{SO}(3)$ at that point. The expanding behavior is speculated to be exponential at early times [10] and to turn into a power law at later times [11] based on results obtained by Monte Carlo simulation of simplified models. Classical solutions, which may describe the expanding behavior at even later times, are also discussed $[12,13]$. These results are encouraging since they seem to suggest that the Lorentzian version of the type IIB matrix model correctly describes the history of our Universe as it should for a nonperturbative formulation of superstring theory. See refs. [14-23] for closely related work in this direction.

In this paper we investigate the effects of the infrared (IR) cutoffs, which are inevitably introduced in the Lorentzian type IIB matrix model. For that purpose, we generalize the form of the IR cutoffs as $\operatorname{tr}\left\{\left(A_{0}\right)^{2}\right\}^{p}$ and $\operatorname{tr} Q^{p}$ with $Q=\sum_{i=1}^{9}\left(A_{i}\right)^{2}$, where $p$ is a real positive parameter. The previous choice corresponds to the $p=1$ case, and in the $p \rightarrow \infty$ limit, the cutoffs constrain only the largest eigenvalues of $\left(A_{0}\right)^{2}$ and $Q$. We first perform Monte Carlo simulation of a simplified model for $1.0 \leq p \leq 1.5$, and find that the results become universal for $p>p_{\text {cr }}$, where $p_{\text {cr }}=1.2 \sim 1.3$. The previous results with $p=1$ agree with this universal behavior qualitatively but not quantitatively. Some preliminary results have been reported in our proceedings article [24].

The universality observed here suggests that the effects of the IR cutoffs disappear in the infinite-volume limit for $p>p_{\text {cr. }}$. In order to clarify this possibility, we consider the Schwinger-Dyson equations (SDE) and calculate each term by Monte Carlo simulation of the simplified model for $p=0.5,1.0$ and 1.5. We find for $p=1.5$ that the terms arising from the IR cutoffs indeed decrease in magnitude compared with the other terms in the SDE as the volume is increased. This is not the case for $p=0.5$ and 1.0.

On the other hand, when $p$ becomes as large as 2.0, we find that large- $N$ scaling behaviors do not show up, which implies that we cannot take a sensible large- $N$ limit unlike the cases with $p \leq 1.5$. This has something to do with the fact that the number of eigenvalues $\alpha_{i}$ of $A_{0}$ that correspond to the time region in which the spontaneous symmetry breaking occurs does not increase with $N$ for $p=2.0$. Interestingly, for $p=2.0$, we observe a $(5+1)$ d structure instead of a $(3+1)$ d structure observed for $p \leq 1.5$.

Thus we conclude that there is a certain range of $p$ in which a universal large- $N$ limit can be taken. Within this range of $p$, the dynamical space-time dimensionality turns out to be $(3+1)$, at least in the simplified model.

The rest of this paper is organized as follows. In section 2 we introduce the Lorentzian type IIB matrix model with a generalized form of the IR cutoffs including the arbitrary parameter $p$. In section 3 we explain the simplification of the model we adopt, and present the results of Monte Carlo simulation for $p \leq 1.5$. In particular, we show that a universal 
behavior is obtained when $p$ is larger than some critical value. In section 4 we investigate the IR cutoff effects by calculating each term in the SDE by Monte Carlo simulation. In section 5 we show that large- $N$ scaling behaviors do not show up for $p$ as large as 2.0. Section 6 is devoted to a summary and discussions.

\section{The Lorentzian type IIB matrix model with generalized IR cutoffs}

The action of the type IIB matrix model is given by [1]

$$
\begin{aligned}
S & =S_{\mathrm{b}}+S_{\mathrm{f}}, \\
S_{\mathrm{b}} & =-\frac{1}{4 g^{2}} \operatorname{Tr}\left(\left[A_{\mu}, A_{\nu}\right]\left[A^{\mu}, A^{\nu}\right]\right), \\
S_{\mathrm{f}} & =-\frac{1}{2 g^{2}} \operatorname{Tr}\left(\Psi_{\alpha}\left(\mathcal{C} \Gamma^{\mu}\right)_{\alpha \beta}\left[A_{\mu}, \Psi_{\beta}\right]\right),
\end{aligned}
$$

where $A_{\mu}(\mu=0, \ldots, 9)$ and $\Psi_{\alpha}(\alpha=1, \ldots, 16)$ are bosonic and fermionic $N \times N$ matrices, respectively, both of which are traceless and Hermitian. The indices $\mu$ and $\nu$ are contracted using the Lorentzian metric $\eta_{\mu \nu}=\operatorname{diag}(-1,1, \ldots, 1)$, whereas the $16 \times 16$ matrices $\Gamma^{\mu}$ and $\mathcal{C}$ are gamma-matrices and the charge conjugation matrix, respectively, after the Weyl projection in (9+1)-dimensions. The model has manifest (9+1)-dimensional Lorentz symmetry, where $A_{\mu}$ and $\Psi_{\alpha}$ transform as a vector and a Majorana-Weyl spinor, respectively. The "coupling constant" $g$ is merely a scale parameter since it can be absorbed by an appropriate rescaling of $A_{\mu}$ and $\Psi_{\alpha}$. The Euclidean version can be obtained by making a "Wick rotation" $A_{0}=i A_{10}$, where $A_{10}$ is supposed to be Hermitian.

The partition function for the Lorentzian version is proposed in ref. [9] as

$$
Z=\int d A d \Psi e^{i S}
$$

with the action (2.1). The " $i$ " in front of the action is motivated from the fact that the string world-sheet metric should also have a Lorentzian signature. By integrating out the fermionic matrices, we obtain the Pfaffian

$$
\int d \Psi e^{i S_{\mathrm{f}}}=\operatorname{Pf} \mathcal{M}(A)
$$

which is real unlike in the Euclidean case [25]. Note also that the bosonic action (2.2) can be written as

$$
S_{\mathrm{b}}=\frac{1}{4 g^{2}} \operatorname{Tr}\left(F_{\mu \nu} F^{\mu \nu}\right)=\frac{1}{4 g^{2}}\left\{-2 \operatorname{Tr}\left(F_{0 i}\right)^{2}+\operatorname{Tr}\left(F_{i j}\right)^{2}\right\}
$$

where we have introduced the Hermitian matrices $F_{\mu \nu}=i\left[A_{\mu}, A_{\nu}\right]$. Since the two terms in the last expression of eq. (2.6) have opposite signs, $S_{\mathrm{b}}$ is not positive semi-definite, which makes the partition function (2.4) divergent. Let us recall that in the Euclidean case, $S_{\mathrm{b}}$ is positive semi-definite, and the partition function is finite $[5,6]$. 
In order to make the partition function (2.4) finite, we introduce IR cutoffs in both the temporal and spatial directions as ${ }^{1}$

$$
\begin{aligned}
& \frac{1}{N} \operatorname{Tr}\left\{\left(A_{0}\right)^{2}\right\}^{p} \leq \kappa^{p} \frac{1}{N} \operatorname{Tr}\left\{\left(A_{i}\right)^{2}\right\}^{p}, \\
& \frac{1}{N} \operatorname{Tr}\left\{\left(A_{i}\right)^{2}\right\}^{p} \leq L^{2 p},
\end{aligned}
$$

which generalizes the original one adopted in ref. [9] corresponding to the $p=1$ case. In what follows, we set $L=1$ without loss of generality. After some manipulation and rescaling of $A_{\mu}$, we can rewrite the partition function (2.4) as [9] (See appendix A of ref. [10] for a refined argument.)

$$
Z=\int d A \operatorname{Pf} \mathcal{M}(A) \delta\left(\frac{1}{N} \operatorname{Tr}\left(F_{\mu \nu} F^{\mu \nu}\right)\right) \delta\left(\frac{1}{N} \operatorname{Tr}\left\{\left(A_{i}\right)^{2}\right\}^{p}-1\right) \theta\left(\kappa^{p}-\frac{1}{N} \operatorname{Tr}\left\{\left(A_{0}\right)^{2}\right\}^{p}\right),
$$

where $\theta(x)$ is the Heaviside step function. This form allows us to perform Monte Carlo simulation without the sign problem unlike in the Euclidean model. ${ }^{2}$

A peculiar feature of the Lorentzian version of the type IIB matrix model is that one can extract the "real-time dynamics" by identifying the eigenvalues of the temporal matrix $A_{0}$ as representing the time [9]. For that purpose we use the $\mathrm{SU}(N)$ symmetry of the model to diagonalize the temporal matrix $A_{0}$ as

$$
A_{0}=\operatorname{diag}\left(\alpha_{1}, \ldots, \alpha_{N}\right), \quad \text { where } \alpha_{1}<\cdots<\alpha_{N} .
$$

In this basis, the spatial matrices $A_{i}$ generated by the Monte Carlo simulation of (2.9) turn out to have an approximate band-diagonal structure. More precisely, there exists some integer $n$ such that the elements of spatial matrices $\left(A_{i}\right)_{a b}$ for $|a-b| \geq n$ are much smaller than those for $|a-b|<n$. Based on this observation, we may naturally consider $n \times n$ matrices

$$
\left(\bar{A}_{i}\right)_{I J}(t) \equiv\left(A_{i}\right)_{\nu+I, \nu+J}
$$

as representing the state of the $9 \mathrm{~d}$ space at time $t$ defined by

$$
t=\frac{1}{n} \sum_{I=1}^{n} \alpha_{\nu+I}
$$

where $I, J=1, \ldots, n$ and $\nu=0,1, \ldots, N-n$. For example, we can define the extent of space at time $t$ as

$$
R^{2}(t)=\left\langle\frac{1}{n} \operatorname{tr} \sum_{i}\left(\bar{A}_{i}(t)\right)^{2}\right\rangle,
$$

\footnotetext{
${ }^{1}$ One might be tempted to introduce a Lorentz invariant IR cutoff of the form $\frac{1}{N} \operatorname{Tr}\left(A_{\mu} A^{\mu}\right)^{p} \leq L^{2 p}$. This does not work, however, because $A_{\mu} A^{\mu}=-\left(A_{0}\right)^{2}+\left(A_{i}\right)^{2}$ can be small in magnitude due to cancellations between the two terms.

${ }^{2}$ Strictly speaking, the Pfaffian Pf $\mathcal{M}$ in (2.9) can change its sign, but configurations with positive Pfaffian dominate at large $N$.
} 
where the symbol tr represents a trace over the $n \times n$ block. We also define the "moment of inertia tensor"

$$
T_{i j}(t)=\frac{1}{n} \operatorname{tr}\left(\bar{A}_{i}(t) \bar{A}_{j}(t)\right)
$$

which is a $9 \times 9$ real symmetric matrix. The eigenvalues of $T_{i j}(t)$, which we denote by $\lambda_{i}(t)$ with the order

$$
\lambda_{1}(t)>\lambda_{2}(t)>\cdots>\lambda_{9}(t)
$$

represent the spatial extent in each of the nine directions at time $t$. The block size $n$ used in calculating quantities such as (2.13) and (2.14) by Monte Carlo simulation is determined as described in section 5 of ref. [11].

In actual simulation, it is convenient to "gauge fix" the $\mathrm{SU}(N)$ symmetry by the condition (2.10). The usual Fadeev-Popov procedure for the gauge fixing implies that the integration $\int d A_{0}$ in (2.9) should be replaced by $\int \prod_{k=1}^{N} d \alpha_{k} \Delta(\alpha)^{2}$, where

$$
\Delta(\alpha) \equiv \prod_{a>b}^{N}\left(\alpha_{a}-\alpha_{b}\right)
$$

is the van der Monde determinant. The delta functions and the step function in (2.9) are replaced by the Gaussian-type potentials in the action given by

$$
\begin{aligned}
S^{(C)} & =\frac{1}{2} \gamma^{(C)} N^{2}\left(\frac{1}{N} \operatorname{Tr}\left(F_{\mu \nu} F^{\mu \nu}\right)\right)^{2}, \\
S^{(L)} & =\frac{1}{2} \gamma^{(L)} N^{2}\left(\frac{1}{N} \operatorname{Tr}\left[\left\{\left(A_{i}\right)^{2}\right\}^{p}\right]-1\right)^{2}, \\
S^{(\kappa)} & = \begin{cases}\frac{1}{2} \gamma^{(\kappa)} N^{2}\left(\frac{1}{N} \operatorname{Tr}\left[\left\{\left(A_{0}\right)^{2}\right\}^{p}\right]-\kappa^{p}\right)^{2} & \text { for } \frac{1}{N} \operatorname{Tr}\left[\left\{\left(A_{0}\right)^{2}\right\}^{p}\right]>\kappa^{p}, \\
0 & \text { otherwise, }\end{cases}
\end{aligned}
$$

where the coefficients $\gamma^{(C)}, \gamma^{(L)}$ and $\gamma^{(\kappa)}$ are taken to be large enough to make the generated configurations satisfy the constraints with good accuracy. Thus, the partition function (2.9) is replaced by ${ }^{3}$

$$
Z=\int \prod_{i=1}^{9} d A_{i} \prod_{k=1}^{N} d \alpha_{k} \Delta(\alpha)^{2} \operatorname{Pf} \mathcal{M}(A) e^{-\left(S^{(C)}+S^{(L)}+S^{(\kappa)}\right)} .
$$

This model can be investigated by Monte Carlo simulation as described in appendix B of ref. [10] for $p=1$.

\footnotetext{
${ }^{3}$ As a yet another technical detail, we use a potential for stabilizing the peak of $R^{2}(t)$ defined by $(2.13)$, whose position would otherwise fluctuate slowly as the simulation proceeds. See appendix B of ref. [10] for the details. This is done just for the sake of effective measurements, and it does not affect the properties of the model. For instance, we have explicitly checked that a term that appears from this peak-stabilizing potential is negligible compared with the other terms in the SDE investigated in section 4.
} 

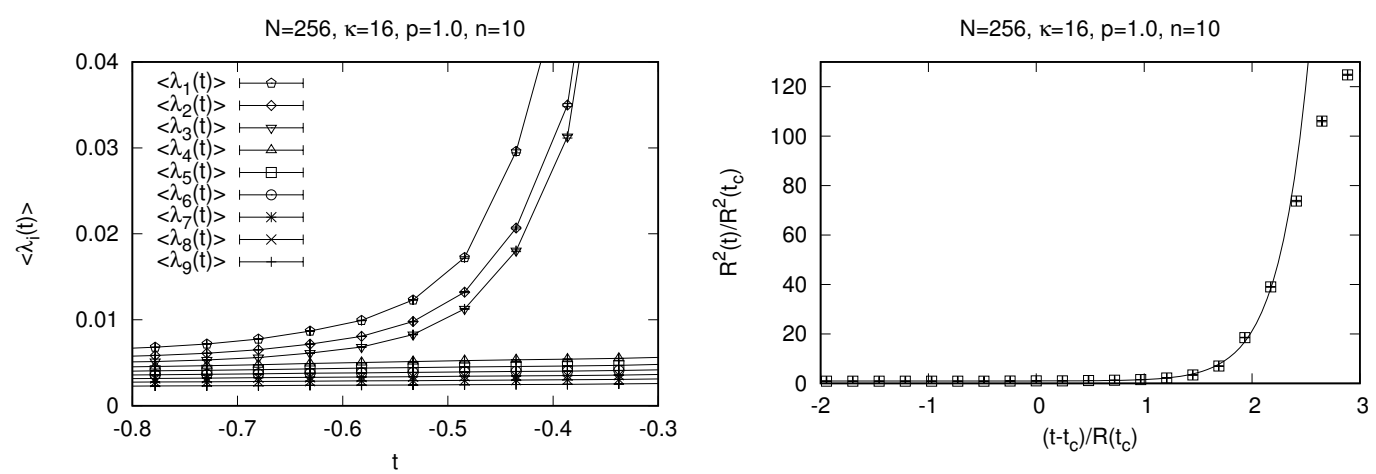

Figure 1. (Left) The expectation values of the eigenvalues $\lambda_{i}(t)$ of $T_{i j}(t)$ are plotted against $t$ for $p=1$ with $N=256$ and $\kappa=16$, where we use the block size $n=10$. The lines are drawn to guide the eye. (Right) The extent of space $R^{2}(t)$ normalized by $R^{2}\left(t_{\mathrm{c}}\right)$ is plotted against $x=\left(t-t_{\mathrm{c}}\right) / R\left(t_{\mathrm{c}}\right)$ for the same set of parameters. The solid line is a fit to the exponential behavior $f(x)=a+(1-a) \exp (b x)$ with a constraint $f(0)=1$, where $a=0.983(3)$ and $b=3.56(11)$.

\section{$3 \quad$ Universality in the results for various $p$}

Let us investigate how the results of the model (2.20) depend on the parameter $p$ introduced in the cutoffs (2.7) and (2.8). Here, we adopt a simplification [10], which amounts to replacing the Pfaffian in $(2.20)$ as

$$
\operatorname{Pf} \mathcal{M}(A) \Longrightarrow \Delta^{16}(\alpha)
$$

where $\Delta(\alpha)$ is the van der Monde determinant defined in (2.16). This simplification occurs when one omits the dependence of $\operatorname{Pf} \mathcal{M}(A)$ on the spatial matrices $A_{i}$, which makes sense at early times, where the expansion of space has not proceeded much. The partition function of the simplified model is given by

$$
Z=\int \prod_{i=1}^{9} d A_{i} \prod_{k=1}^{N} d \alpha_{k} \Delta(\alpha)^{18} e^{-\left(S^{(C)}+S^{(L)}+S^{(\kappa)}\right)},
$$

and the computational cost is considerably reduced from that of the original model (2.20).

Let us first consider the case in which $p=1$ is used for the IR cutoffs (2.7) and (2.8) as is done in all the previous work. Figure 1 (Left) shows the expectation values of the eigenvalues $\lambda_{i}(t)$ of $T_{i j}(t)$ defined by (2.14) as a function of $t$ for the simplified model with $N=256$ and $\kappa=16$. We find that the $\mathrm{SO}(9)$ symmetry is spontaneously broken down to $\mathrm{SO}(3)$ at a critical time $t_{\mathrm{c}}=-0.68014(7)$. (Precise definition of $t_{\mathrm{c}}$ is given in section 3 of ref. [10].) Since the extent of space $R^{2}\left(t_{\mathrm{c}}\right)=0.04099(4)$ at the critical time $t_{\mathrm{c}}$ is a physical quantity which is dimensionful, we use it to fix the scale of the system. In figure 1 (Right), we plot the extent of space $R^{2}(t)$ normalized by $R^{2}\left(t_{\mathrm{c}}\right)$ against $x=\left(t-t_{\mathrm{c}}\right) / R\left(t_{\mathrm{c}}\right)$ for the same $N$ and $\kappa$. The result can be nicely fitted to an exponential function. (Note that the data points at late times are affected by finite $N$ effects as one can see from the large- $N$ scaling behaviors in figure 3 below.) Similar behaviors were observed previously in the $(5+1)$ d version of the simplified model with the matrix size $N \leq 64$ [10]. 


\begin{tabular}{|c|c|c|c|c|c|c|c|}
\hline$p$ & $N$ & $\kappa$ & $n$ & $t_{\mathrm{c}}$ & $R^{2}\left(t_{\mathrm{c}}\right)$ & $a$ & $b$ \\
\hline 1.0 & 256 & 16 & 10 & $-0.68014(7)$ & $0.04099(04)$ & $0.983(03)$ & $3.56(11)$ \\
\hline 1.1 & 256 & 16 & 6 & $-0.39307(6)$ & $0.03213(14)$ & $0.961(18)$ & $5.36(39)$ \\
\hline 1.2 & 256 & 16 & 6 & $-0.34441(6)$ & $0.02904(16)$ & $0.976(12)$ & $6.82(53)$ \\
\hline 1.3 & 256 & 16 & 6 & $-0.29213(8)$ & $0.03055(11)$ & $0.940(12)$ & $8.10(28)$ \\
\hline 1.4 & 256 & 16 & 6 & $-0.23933(8)$ & $0.02940(19)$ & $0.944(27)$ & $8.07(63)$ \\
\hline 1.5 & 256 & 16 & 6 & $-0.23593(7)$ & $0.02579(02)$ & $0.950(11)$ & $8.24(30)$ \\
\hline
\end{tabular}

Table 1. The block size $n$, the critical time $t_{\mathrm{c}}$ and the extent of space $R^{2}\left(t_{\mathrm{c}}\right)$ at the critical time, which are used to make the plots in figure 2 , are given for each $p$. We also present the values of $a$ and $b$ obtained by fitting $R^{2}(t) / R^{2}\left(t_{\mathrm{c}}\right)$ to $f(x)=a+(1-a) \exp (b x)$ with $x=\left(t-t_{\mathrm{c}}\right) / R\left(t_{\mathrm{c}}\right)$ for each $p$.

Next we show our results for $p \neq 1$. In figure 2 we plot the extent of space $R^{2}(t)$ normalized by $R^{2}\left(t_{\mathrm{c}}\right)$ against $x=\left(t-t_{\mathrm{c}}\right) / R\left(t_{\mathrm{c}}\right)$ for various values of $p$ within $1 \leq p \leq$ 1.5. While the results exhibit certain $p$-dependence, qualitative behaviors such as the exponential expansion remain the same as those for $p=1.0$. We have also confirmed that only three directions start to expand at some critical time for the values of $p$ within this region. What is most remarkable in these plots is that the data points for $1.3 \leq p \leq 1.5$ lie on a single curve except for the region of $t$ in which $R^{2}(t)$ approaches its maximum. This universality suggests that the IR cutoffs are not affecting the results for these values of $p$ except near the spatial "boundary", where the cutoff effects should, of course, be visible.

In the next section, we examine this interpretation directly by investigating the IR cutoff effects through the SDE. In particular, we show that the IR cutoff effects actually decrease in magnitude for large enough $p$ as we take the infinite-volume limit. In the remainder of this section, we discuss how we take this limit. First we define the "volume" $\Delta$ and the "lattice spacing" $\varepsilon$ in the temporal direction by

$$
\Delta \equiv \frac{t_{\text {peak }}-t_{\mathrm{c}}}{R\left(t_{\mathrm{c}}\right)}, \quad \varepsilon=\frac{\Delta}{\nu},
$$

where $t_{\text {peak }}$ represents the time $t$ at which $R^{2}(t)$ becomes maximum, and $\nu$ is the number of data points within $\Delta$. The infinite-volume limit corresponds to increasing $\Delta$ with fixed $\varepsilon$, while the continuum limit corresponds to decreasing $\varepsilon$ with fixed $\Delta$. By tuning the cutoff parameter $\kappa$ as one increases $N$, one can take these limits separately or simultaneously.

When we investigate the SDE in section 4, we need to take the infinite-volume limit since the IR cutoff effects are expected to disappear in that limit. For that purpose, we tune the cutoff parameter $\kappa$ as we increase $N$ for each $p$ so that the lattice spacing is kept almost constant in $N$. In table 2 , we show the values of $\kappa$ thus obtained together with the lattice spacing $\varepsilon$ and the volume $\Delta$ measured using (3.3). In figure 3, we plot the extent of space $R^{2}(t)$ normalized by $R^{2}\left(t_{\mathrm{c}}\right)$ against $x=\left(t-t_{\mathrm{c}}\right) / R\left(t_{\mathrm{c}}\right)$ for the set of parameters given in table 2. We find that the horizontal distance of the data points is almost independent of $N$, while the extent in the temporal and spatial directions grows with $N$.

Note also that figure 3 exhibits a scaling region in which the data for different $N$ lie on top of each other. In making these plots, we have shifted the value of $t_{\mathrm{c}}$ slightly so 

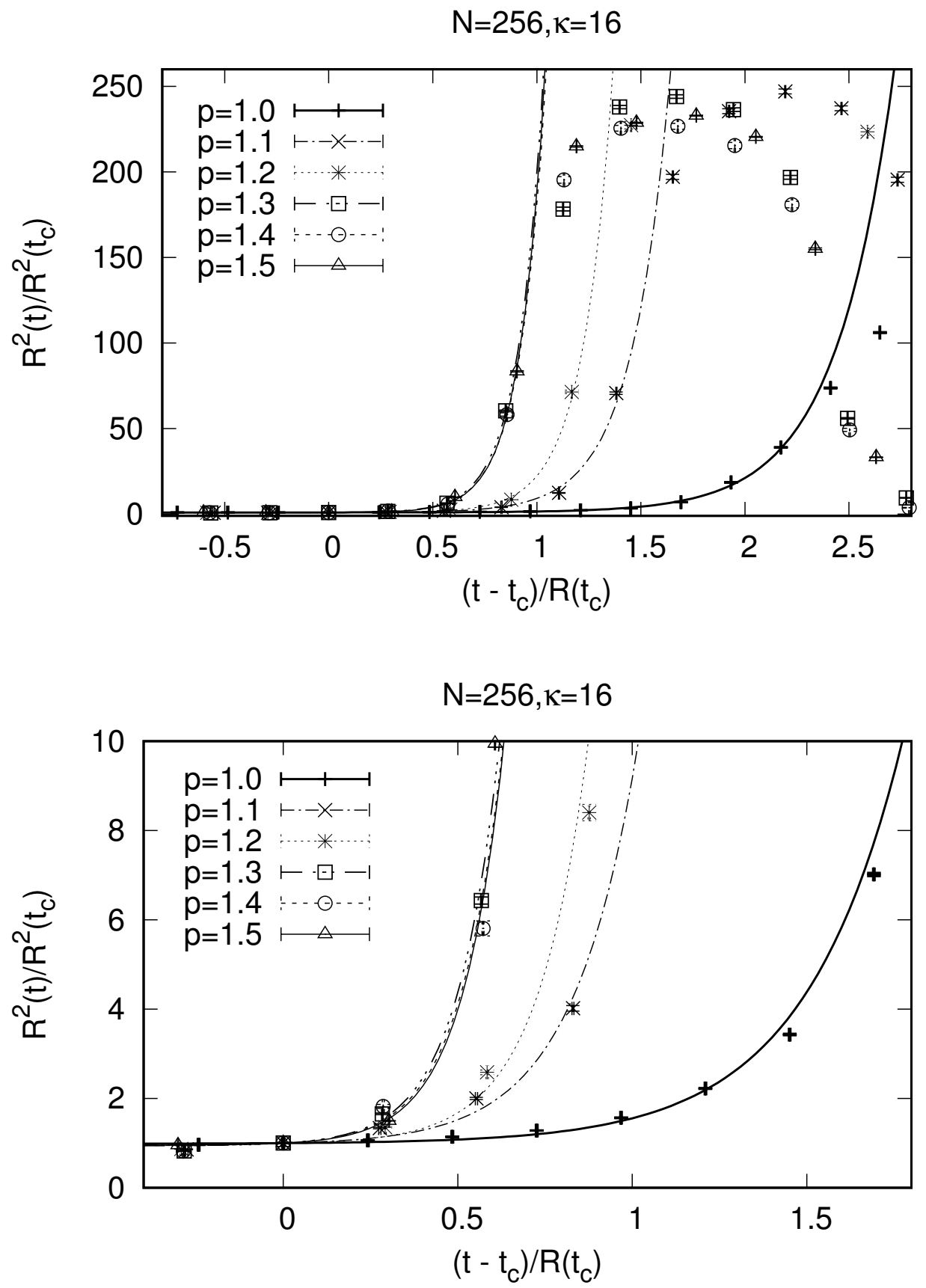

Figure 2. (Top) The extent of space $R^{2}(t)$ normalized by $R^{2}\left(t_{\mathrm{c}}\right)$ is plotted against $x=$ $\left(t-t_{\mathrm{c}}\right) / R\left(t_{\mathrm{c}}\right)$ for $1.0 \leq p \leq 1.5$ with $N=256$ and $\kappa=16$. The parameters used to make these plots are given in table 1 . The lines are fits to $R^{2}(t) / R^{2}\left(t_{\mathrm{c}}\right)=a+(1-a) \exp (b x)$. The values of the fitting parameters $a$ and $b$ obtained by the fits are also presented in table 1. (Bottom) Zoom up of the plot at the top. 

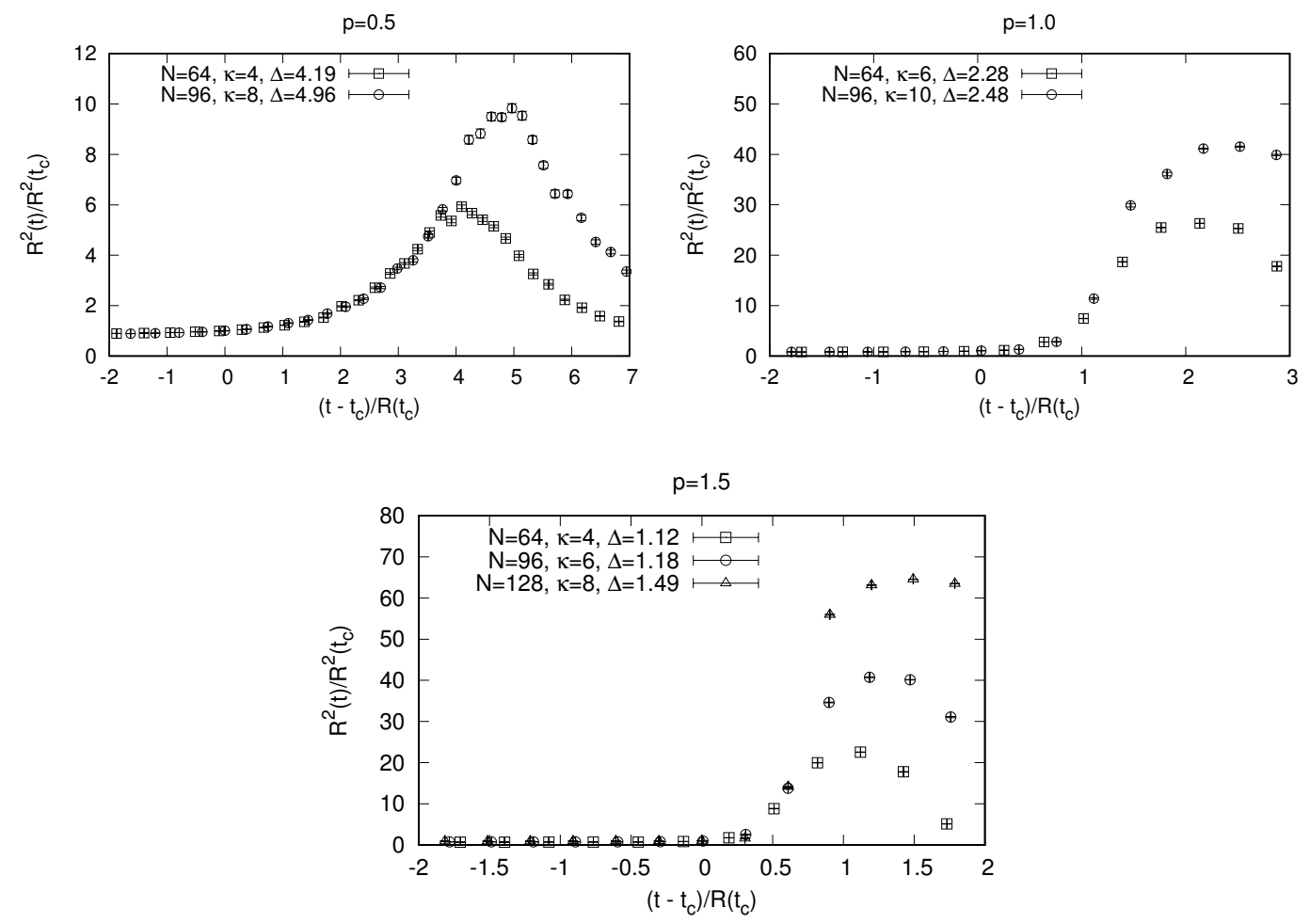

Figure 3. The extent of space $R^{2}(t)$ normalized by $R^{2}\left(t_{\mathrm{c}}\right)$ is plotted against $x=\left(t-t_{\mathrm{c}}\right) / R\left(t_{\mathrm{c}}\right)$ for $p=0.5$ (Top-Left), $p=1.0$ (Top-Right) and $p=1.5$ (Bottom). The parameters $N$ and $\kappa$ are chosen as in table 2 for each $p$ so that the lattice spacing $\varepsilon$ is kept almost constant in $N$, while the volume $\Delta$ in the temporal direction increases with $N$.

\begin{tabular}{|c|c|c|c|c|c|c|c|}
\hline$p$ & $N$ & $\kappa$ & $n$ & $t_{\mathrm{c}}$ & $R^{2}\left(t_{\mathrm{c}}\right)$ & $\varepsilon$ & $\Delta$ \\
\hline 0.5 & 64 & 4 & 8 & $-1.27274(45)$ & $0.15612(90)$ & 0.28 & 4.19 \\
\hline 0.5 & 96 & 8 & 8 & $-1.54629(53)$ & $0.09735(66)$ & 0.27 & 4.96 \\
\hline 1.0 & 64 & 6 & 6 & $-0.71603(18)$ & $0.09398(16)$ & 0.38 & 2.28 \\
\hline 1.0 & 96 & 10 & 8 & $-0.83132(75)$ & $0.07762(39)$ & 0.35 & 2.48 \\
\hline 1.5 & 64 & 4 & 4 & $-0.38586(16)$ & $0.07797(18)$ & 0.312 & 1.12 \\
\hline 1.5 & 96 & 6 & 5 & $-0.35286(14)$ & $0.06658(26)$ & 0.294 & 1.18 \\
\hline 1.5 & 128 & 8 & 6 & $-0.33829(19)$ & $0.05349(14)$ & 0.298 & 1.49 \\
\hline
\end{tabular}

Table 2. The set of parameters $N$ and $\kappa$ chosen for each $p$ in such a way that the lattice spacing $\varepsilon$ is kept almost constant in $N$, while the volume $\Delta$ in the temporal direction increases with $N$. The block size $n$, the critical time $t_{\mathrm{c}}$ and the extent of space $R^{2}\left(t_{\mathrm{c}}\right)$ at the critical time are also given.

that the observed scaling behavior is optimized, which is legitimate taking into account the ambiguity in the definition of $t_{\mathrm{c}}$ at finite $N$. Similar shifts are used also in figure 5 , figure 6 (Top-Left), figure 6 (Top-Right) and figure 9, where we discuss large- $N$ asymptotic behaviors. 


\section{Probing the IR cutoff effects by the SDE}

As we mentioned above, the universal behavior observed in figure 2 suggests that the effects of the IR cutoffs (2.7) and (2.8) vanish in the infinite-volume limit for sufficiently large $p$. In order to clarify this possibility, we investigate the effects directly by using the SDE.

Here we rewrite the partition function (3.2) as

$$
\begin{aligned}
& Z=\int d A d \alpha e^{-S}, \\
& S=S^{(C)}+S^{(L)}+S^{(\kappa)}+S^{(\alpha)},
\end{aligned}
$$

where $S^{(C)}, S^{(L)}$ and $S^{(\kappa)}$ are defined by $(2.17),(2.18)$ and $(2.19)$, respectively, and $S^{(\alpha)}$ is defined by

$$
S^{(\alpha)}=-18 \sum_{a>b} \ln \left(\alpha_{a}-\alpha_{b}\right)
$$

Let us then consider the SDE

$$
\begin{aligned}
\frac{1}{Z} \int d A d \alpha \frac{\partial}{\partial \alpha_{b}}\left(\alpha_{a} e^{-S}\right) & =0 \\
\frac{1}{Z} \int d A d \alpha \frac{\partial}{\partial\left(A_{j}\right)_{c d}}\left(\left(A_{i}\right)_{a b} e^{-S}\right) & =0 .
\end{aligned}
$$

Contracting some indices, we obtain the identities

$$
\begin{aligned}
\left\langle\alpha_{a} \frac{\partial S}{\partial \alpha_{b}}\right\rangle & =\delta_{a b}-\frac{1}{N} \\
\frac{1}{9 N} \sum_{i=1}^{9} \sum_{c=1}^{N}\left\langle\left(A_{i}\right)_{a c} \frac{\partial S}{\partial\left(A_{i}\right)_{b c}}\right\rangle & =\left(1-\frac{1}{N^{2}}\right) \delta_{a b},
\end{aligned}
$$

which should be satisfied for each $a$ and $b$. Below we focus on the identities corresponding to the $a=b$ case. Corresponding to the decomposition (4.2) of the action, we obtain

$$
\begin{aligned}
G_{a}^{(C)}+G_{a}^{(\kappa)}+G_{a}^{(\alpha)} & =1-\frac{1}{N}, \\
H_{a}^{(C)}+H_{a}^{(L)} & =1-\frac{1}{N^{2}}
\end{aligned}
$$

where $a=1, \cdots, N$ and we have defined

$$
\begin{gathered}
G_{a}^{(C)} \equiv\left\langle\alpha_{a} \frac{\partial S^{(C)}}{\partial \alpha_{a}}\right\rangle, \quad G_{a}^{(\kappa)} \equiv\left\langle\alpha_{a} \frac{\partial S^{(\kappa)}}{\partial \alpha_{a}}\right\rangle, \quad G_{a}^{(\alpha)} \equiv\left\langle\alpha_{a} \frac{\partial S^{(\alpha)}}{\partial \alpha_{a}}\right\rangle \\
H_{a}^{(C)} \equiv \frac{1}{9 N} \sum_{i=1}^{9} \sum_{c=1}^{N}\left\langle\left(A_{i}\right)_{a c} \frac{\partial S^{(C)}}{\partial\left(A_{i}\right)_{a c}}\right\rangle, \quad H_{a}^{(L)} \equiv \frac{1}{9 N} \sum_{i=1}^{9} \sum_{c=1}^{N}\left\langle\left(A_{i}\right)_{a c} \frac{\partial S^{(L)}}{\partial\left(A_{i}\right)_{a c}}\right\rangle .
\end{gathered}
$$

In figure 4 , we plot $G_{a}^{(C)}, G_{a}^{(\kappa)}, G_{a}^{(\alpha)}$ and their sum against $\left(\alpha_{a}-t_{\mathrm{c}}\right) / R\left(t_{\mathrm{c}}\right)$ for $N=32$ and $\kappa=6$ with $p=0.5,1.0$ and 1.5. We find that the sum of the three terms is constant and agrees with $1-\frac{1}{N}$, which implies that the temporal $\operatorname{SDE}(4.8)$ is satisfied for all $\alpha_{a}$. 

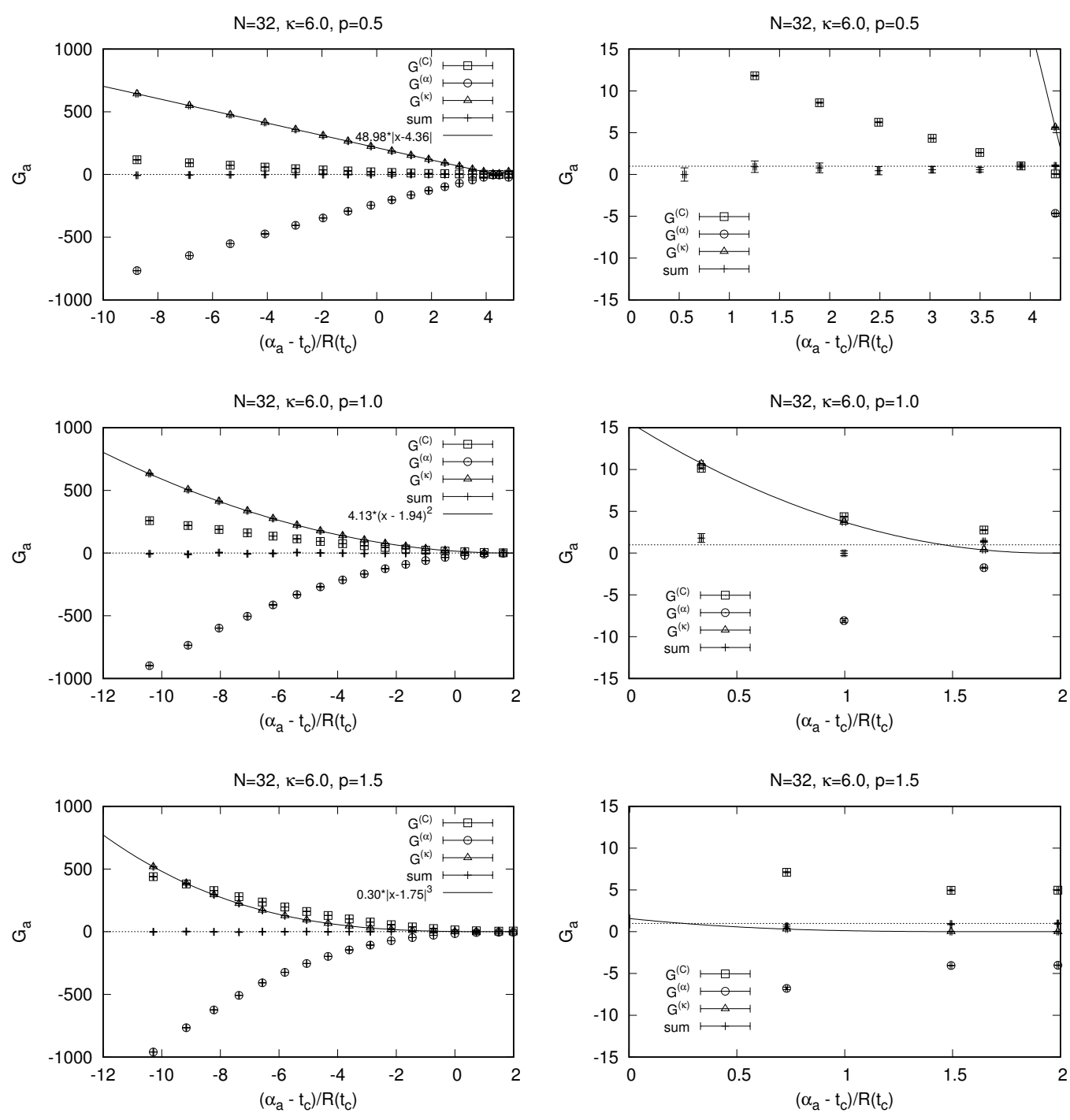

Figure 4. The terms $G_{a}^{(C)}, G_{a}^{(\kappa)}$ and $G_{a}^{(\alpha)}$ in the temporal SDE (4.8) are plotted against $x=$ $\left(\alpha_{a}-t_{\mathrm{c}}\right) / R\left(t_{\mathrm{c}}\right)$ for $p=0.5$ (Top), $p=1.0$ (Middle) and $p=1.5$ (Bottom) with $N=32$ and $\kappa=6$. The plots on the right are zoom up of the plots on the left in the $t \geq 0$ region. We also plot the sum of the three terms, which agrees well with $1-1 / N$ represented by the dotted lines. The solid lines represent fits of $G_{a}^{(\alpha)}$ to the $\left(\alpha_{a}\right)^{2 p}$ behavior.

We also find that $G_{a}^{(\kappa)}$ can be nicely fitted to the $\left(\alpha_{a}\right)^{2 p}$ behavior. This is understandable since $G_{a}^{(\kappa)}$ can be written explicitly as

$$
G_{a}^{(\kappa)}=c\left(\left(\alpha_{a}\right)^{2 p}-\frac{\alpha_{a}}{N} \sum_{b=1}^{N} \alpha_{b}\left(\alpha_{b}\right)^{2(p-1)}\right),
$$

where the coefficient is given as

$$
c=2 p \gamma^{(\kappa)} N\left[\frac{1}{N} \sum_{b=1}^{N}\left(\alpha_{b}^{2}\right)^{p}-\kappa^{p}\right]
$$

and the first term in (4.12) actually dominates. 

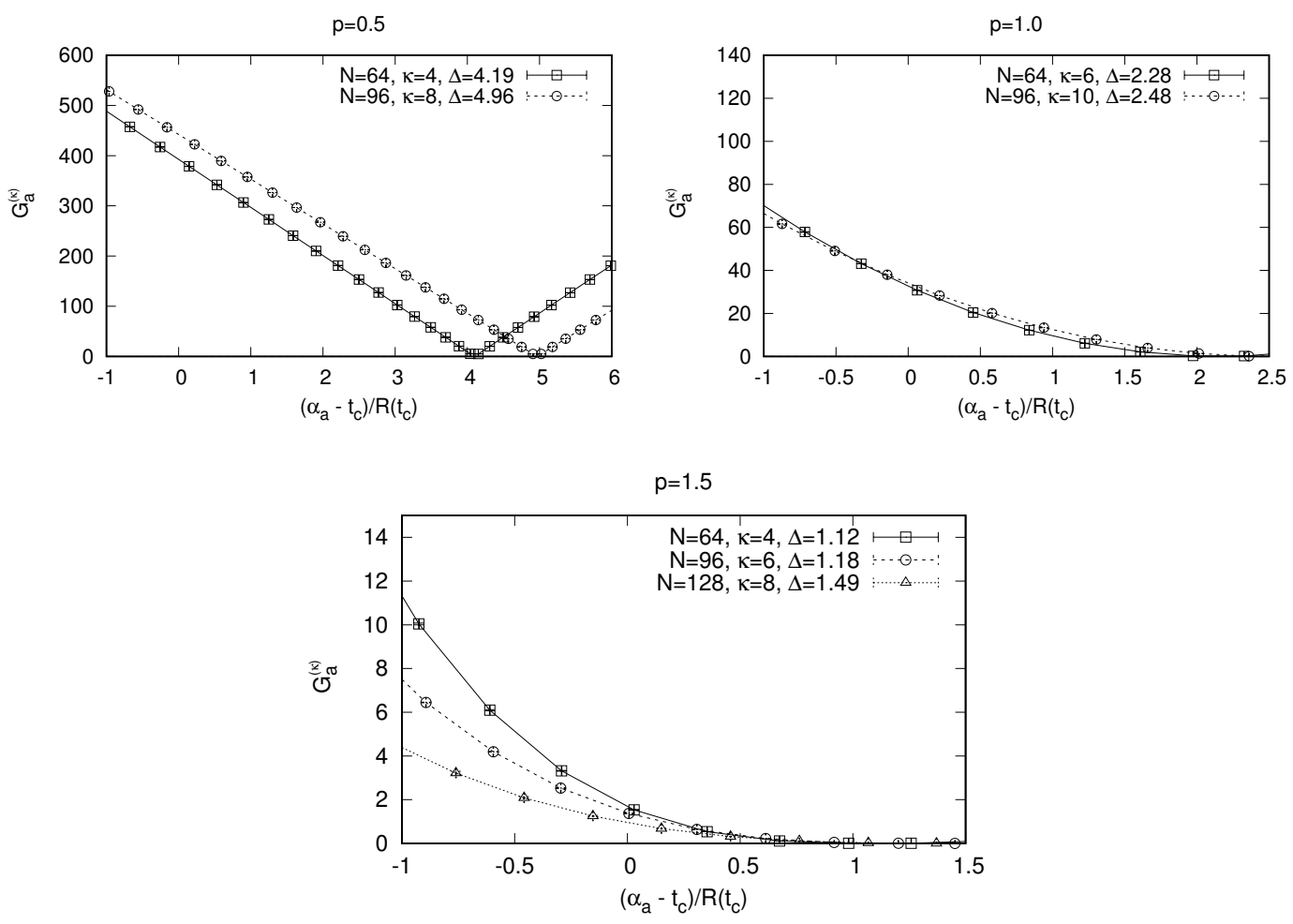

Figure 5. The IR cutoff term $G_{a}^{(\kappa)}$ in the temporal SDE (4.8) is plotted against $x=\left(\alpha_{a}-t_{\mathrm{c}}\right) / R\left(t_{\mathrm{c}}\right)$ for $p=0.5$ (Top-Left), $p=1.0$ (Top-Right) and $p=1.5$ (Bottom) with $\kappa$ and $N$ given in table 2 . The lines are drawn to guide the eye.

From this figure, we find that the effects of the IR cutoffs in the temporal direction represented by $G_{a}^{(\kappa)}$ become large towards the boundary in the temporal direction represented by the left-most point in the plots on the left. However, the IR cutoff effects are suppressed as one goes away from the boundary, in particular for large $p$ as expected from (4.12).

Let us then consider the infinite-volume limit discussed at the end of section 3 and see how the IR cutoff effects behave in that limit. In figure 5 , we plot $G_{a}^{(\kappa)}$ against $x=$ $\left(\alpha_{a}-t_{\mathrm{c}}\right) / R\left(t_{\mathrm{c}}\right)$ for $p=0.5, p=1.0$ and $p=1.5$. The parameters $N$ and $\kappa$ are chosen as in table 2 so that the lattice spacing in the temporal direction is kept almost constant in $N$, while the volume $\Delta$ increases with $N$. We find that $G_{a}^{(\kappa)}$ increases with the volume $\Delta$ for $p=0.5$, whereas it decreases with the volume $\Delta$ for $p=1.5$. For $p=1.0$, the results of $G_{a}^{(\kappa)}$ for different $\Delta$ lie almost on top of each other.

Our results for the SDE in the spatial direction are presented in appendix A, where we find that the term $H_{a}^{(L)}$ in (4.9), which comes from the spatial cutoff, decreases in magnitude for $p=1.5$ as the infinite-volume limit is taken. This is not the case for $p=0.5$ and $p=1.0$.

\section{Absence of large- $N$ scaling behavior for $p=2.0$}

In this section we discuss the results obtained for larger $p$. Here we focus on $p=2.0$, in which case the IR cutoffs in (2.7) and (2.8) involve a term with the same canonical 

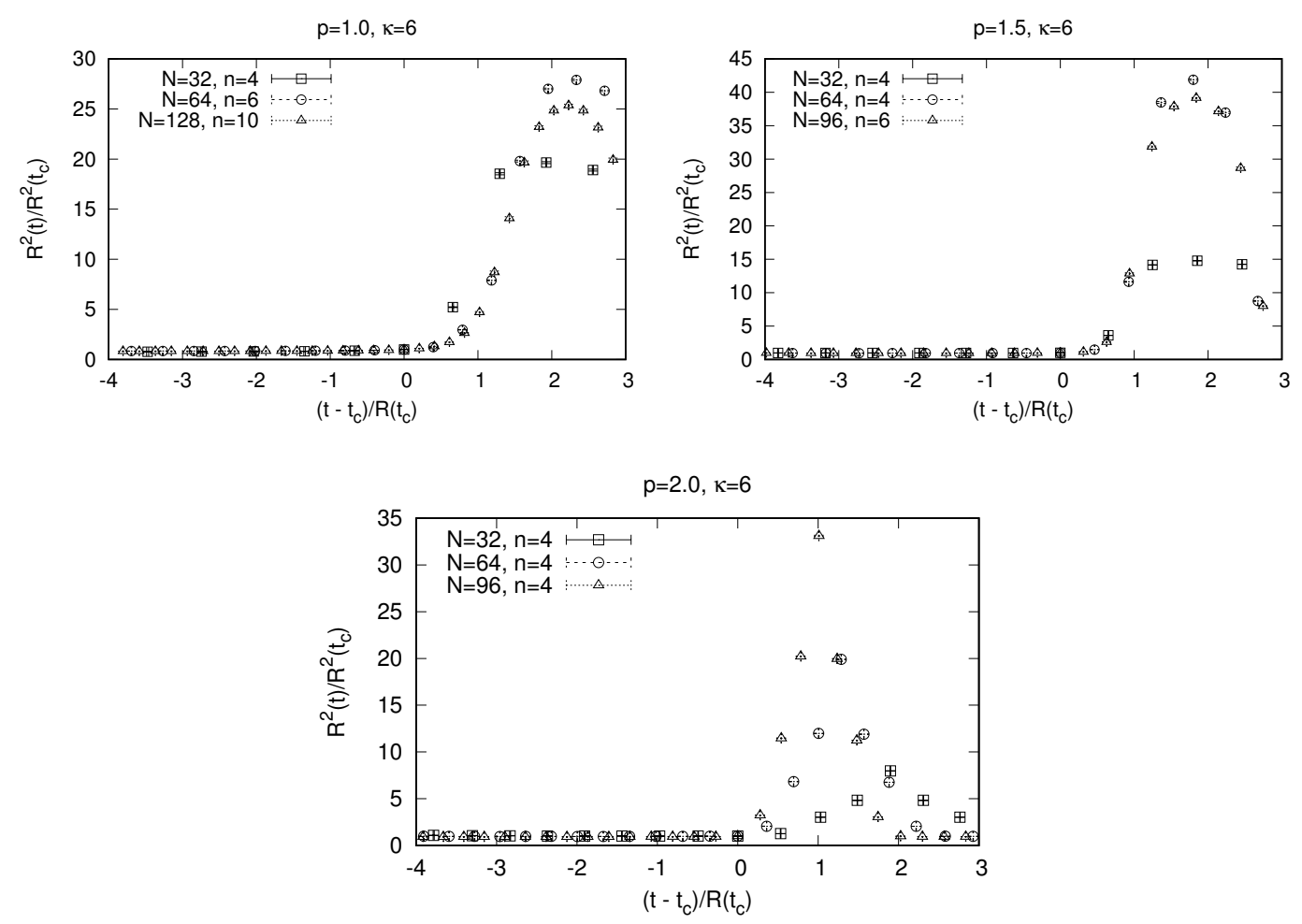

Figure 6. The extent of space $R^{2}(t)$ normalized by $R^{2}\left(t_{\mathrm{c}}\right)$ is plotted against $x=\left(t-t_{\mathrm{c}}\right) / R\left(t_{\mathrm{c}}\right)$ for $\kappa=6$ with $p=1.0$ (Top-Left), $p=1.5$ (Top-Right) and $p=2.0$ (Bottom).

dimension as the bosonic action (2.2). In figure 6 we plot the extent of space $R^{2}(t)$ normalized by $R^{2}\left(t_{\mathrm{c}}\right)$ for $\kappa=6$ with $p=1.0$ (Top-Left), $p=1.5$ (Top-Right) and $p=2.0$ (Bottom). While we observe large- $N$ scaling behaviors for $p=1.0$ and $p=1.5$ as we have already seen in figure 3 , this turns out to be not the case for $p=2.0$. Hence, we cannot take a sensible large- $N$ limit for $p=2.0$. In fact, the number of data points in the region where the spontaneous breaking of $\mathrm{SO}(9)$ symmetry occurs increases with $N$ for $p=1.0$ and $p=1.5$, but not for $p=2.0$, where we have seven data points in the symmetry broken region for all $N$. We consider that this is the reason why large- $N$ scaling behaviors do not show up for $p=2.0$.

Another interesting observation here concerns the dimensionality of the space. In figure 7 we plot the expectation values of the eigenvalues $\lambda_{i}(t)$ of $T_{i j}(t)$ obtained for $p=2.0, N=64$ and $\kappa=6$. We observe five large values near $t=0$, which indicates the emergence of a $5 \mathrm{~d}$ structure. However, we emphasize that this by no means implies that $(5+1)$ d space-time can also appear from the model since one cannot take a sensible large- $N$ limit for $p=2.0$.

\section{Summary}

In this paper, we have addressed an important issue in the Lorentzian type IIB matrix model concerning the IR cutoffs, which are inevitably introduced to make the model well- 


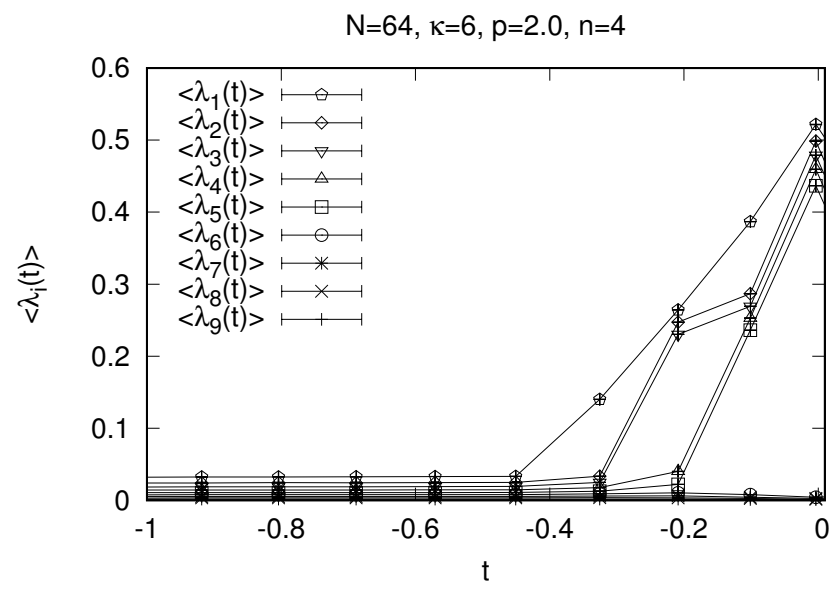

Figure 7. The expectation values of the eigenvalues $\lambda_{i}(t)$ of $T_{i j}(t)$ are plotted against $t$ for $p=2.0$, $N=64$ and $\kappa=6$, where we use the block size $n=4$. The lines are drawn to guide the eye.

defined. In particular, we have generalized the form of the IR cutoffs as (2.7) and (2.8) with a parameter $p$, and performed Monte Carlo simulation of the simplified model for various values of $p$. From the results obtained for $p \leq 1.5$, we observe a universal behavior for $p=1.3,1.4,1.5$ except near the "boundary". This suggests the possibility that the effects of the IR cutoffs vanish in the infinite-volume limit for $p>p_{\mathrm{cr}}$, where $p_{\mathrm{cr}}=1.2 \sim 1.3$. In order to clarify this possibility, we have investigated the effects of the IR cutoffs directly by the SDE. The results show clear tendency that the IR cutoff effects decrease as we take the infinite-volume limit for sufficiently large $p$.

On the other hand, for $p$ as large as 2.0 , we observe that the number of data points in the region with the spontaneous breaking of $\mathrm{SO}(9)$ symmetry does not increase with $N$, and that large- $N$ scaling behaviors do not show up. Combining this with the results obtained for $p \leq 1.5$, we conclude that there exists a finite range of $p$, in which a sensible large- $N$ limit can be taken and the results become independent of $p$. While this range of $p$ does not include the value $p=1.0$ used in the previous work, the qualitative properties of the model such as the dimensionality of the emergent space-time and the exponential expansion remain the same. It is also interesting that a $(5+1) \mathrm{d}$ structure is observed for $p=2.0$, where a sensible large- $N$ limit cannot be taken.

We consider that a similar conclusion holds also in the original Lorentzian type IIB matrix model since the simplified model captures the early time behaviors qualitatively. It is therefore important to study the original model with various $p$ and to identify the region of $p$, in which a universal large- $N$ limit can be taken. We hope to address this issue in future publications.

\section{Acknowledgments}

We thank T. Azuma and S.-W. Kim for their participation at the early stage of this work. We are also grateful to H. Kawai for valuable comments and discussions. This research was supported by MEXT as "Priority Issue on Post-K computer" (Elucidation of the Fun- 

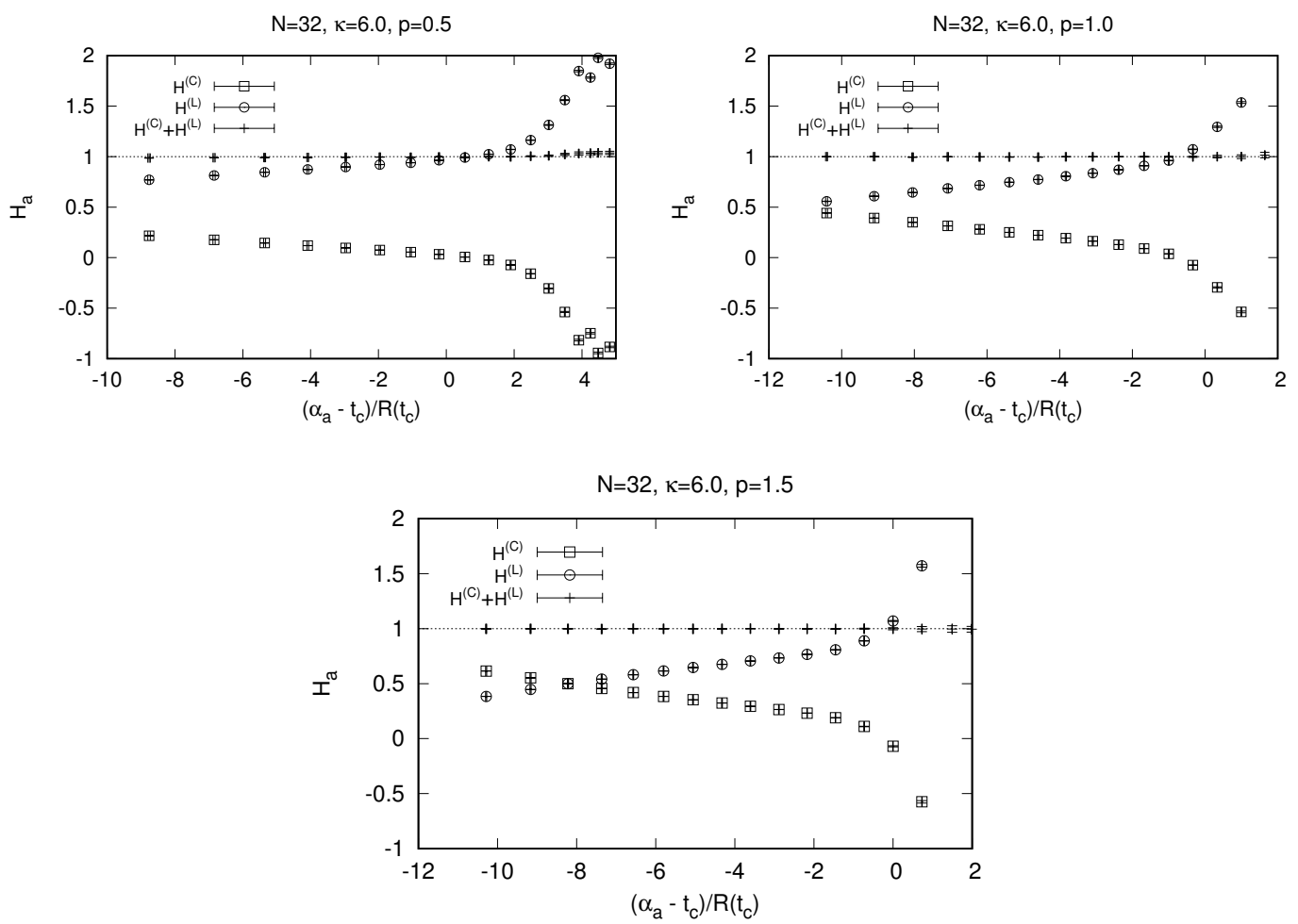

Figure 8. The terms $H_{a}^{(C)}$ and $H_{a}^{(L)}$ in the spatial SDE (4.9) are plotted against $x=$ $\left(\alpha_{a}-t_{\mathrm{c}}\right) / R\left(t_{\mathrm{c}}\right)$ for $p=0.5$ (Top-Left), $p=1.0$ (Top-Right) and $p=1.5$ (Bottom) with $N=32$ and $\kappa=6$. We also plot the sum of the two terms, which agrees well with $1-1 / N^{2}$ represented by the dotted lines.

damental Laws and Evolution of the Universe) and Joint Institute for Computational Fundamental Science (JICFuS). Computations were carried out using computational resources of the K computer provided by the RIKEN Advanced Institute for Computational Science through the HPCI System Research project (Project ID:hp150082). The supercomputer FX10 at University of Tokyo has been used in developing our code for parallel computing. J. N. and A. T. were supported in part by Grant-in-Aid for Scientific Research (No. 23244057 and 15K05046, respectively) from Japan Society for the Promotion of Science.

\section{A Results for the spatial SDE}

In this section, we present our results for the spatial $\operatorname{SDE}(4.9)$. In figure 8, we plot $H_{a}^{(C)}$, $H_{a}^{(L)}$ and their sum against $x=\left(\alpha_{a}-t_{\mathrm{c}}\right) / R\left(t_{\mathrm{c}}\right)$ obtained for $N=32$ and $\kappa=6$ with $p=0.5,1.0$ and 1.5. We find that the spatial SDE (4.9) is actually satisfied for all $\alpha_{a}$. The effects of the IR cutoffs in the spatial direction represented by $H_{a}^{(L)}$ become large towards the "boundary" represented by the right-most point in these plots, where the extent of space $R(t)$ becomes maximum.

In figure 9 , we plot $H_{a}^{(L)}$ against $x=\left(\alpha_{a}-t_{\mathrm{c}}\right) / R\left(t_{\mathrm{c}}\right)$ for $p=0.5, p=1.0$ and $p=1.5$ with the parameters $N$ and $\kappa$ chosen as in table 2. Let us focus on the region in which 

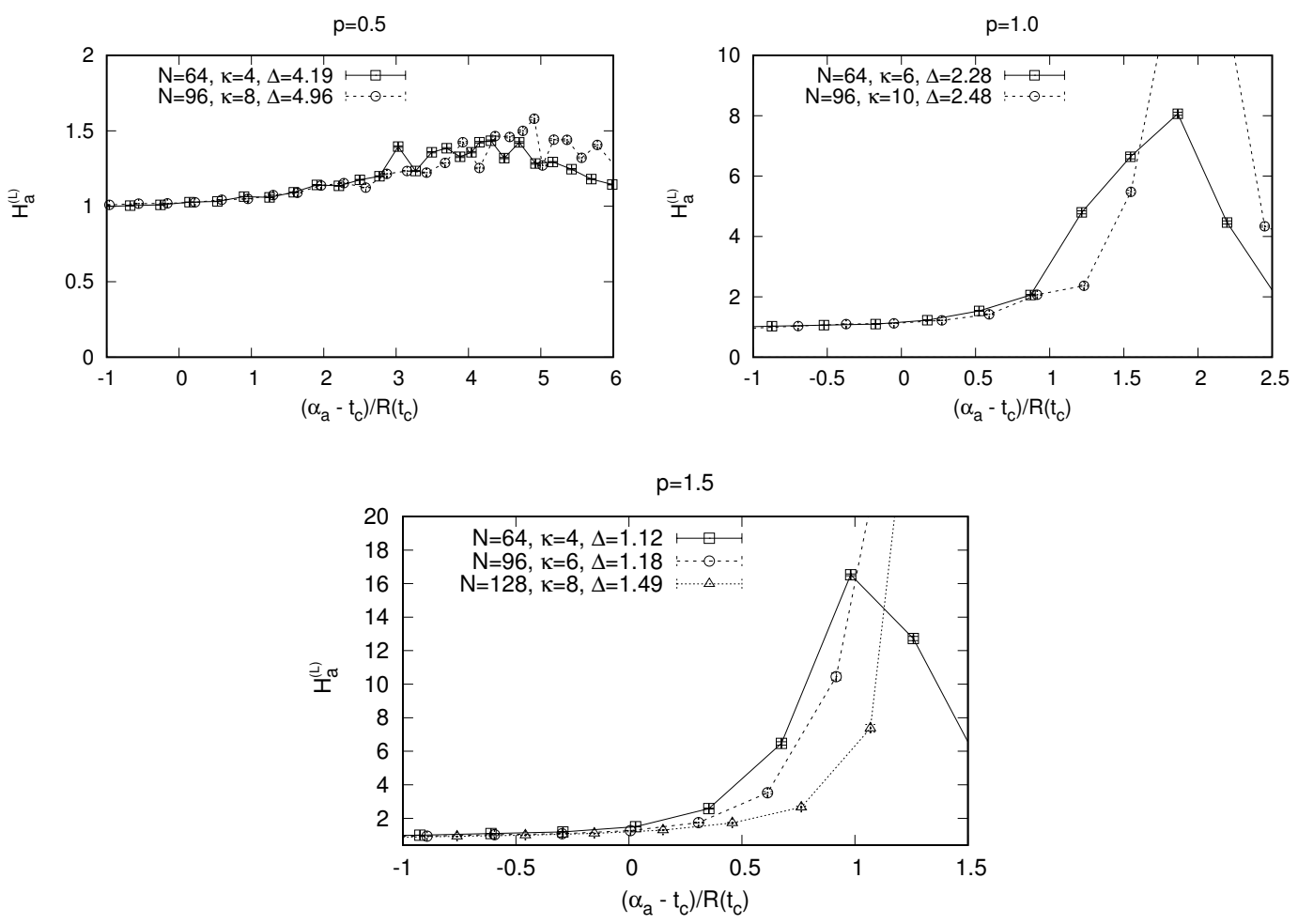

Figure 9. The IR cutoff term $H_{a}^{(L)}$ in the spatial SDE (4.9) is plotted against $x=\left(\alpha_{a}-t_{\mathrm{c}}\right) / R\left(t_{\mathrm{c}}\right)$ for $p=0.5$ (Top-Left), $p=1.0$ (Top-Right) and $p=1.5$ (Bottom) with $\kappa$ and $N$ given in table 2 . The lines are drawn to guide the eye.

we observe scaling behaviors in figure 3 ; namely $x \lesssim 4$ for $p=0.5, x \lesssim 1$ for $p=1.0$ and $x \lesssim 0.6$ for $p=1.5$. In these scaling regions, we find for $p=0.5$ and $p=1.0$ that $H_{a}^{(L)}$ is more or less independent of the volume $\Delta$, whereas for $p=1.5$, we see a clear trend showing that it decreases with the volume $\Delta$.

Open Access. This article is distributed under the terms of the Creative Commons Attribution License (CC-BY 4.0), which permits any use, distribution and reproduction in any medium, provided the original author(s) and source are credited.

\section{References}

[1] N. Ishibashi, H. Kawai, Y. Kitazawa and A. Tsuchiya, A large- $N$ reduced model as superstring, Nucl. Phys. B 498 (1997) 467 [hep-th/9612115] [INSPIRE].

[2] T. Banks, W. Fischler, S.H. Shenker and L. Susskind, $M$ theory as a matrix model: a conjecture, Phys. Rev. D 55 (1997) 5112 [hep-th/9610043] [INSPIRE].

[3] R. Dijkgraaf, E.P. Verlinde and H.L. Verlinde, Matrix string theory, Nucl. Phys. B 500 (1997) 43 [hep-th/9703030] [INSPIRE].

[4] M. Fukuma, H. Kawai, Y. Kitazawa and A. Tsuchiya, String field theory from IIB matrix model, Nucl. Phys. B 510 (1998) 158 [hep-th/9705128] [INSPIRE]. 
[5] W. Krauth, H. Nicolai and M. Staudacher, Monte Carlo approach to M-theory, Phys. Lett. B 431 (1998) 31 [hep-th/9803117] [INSPIRE].

[6] P. Austing and J.F. Wheater, Convergent Yang-Mills matrix theories, JHEP 04 (2001) 019 [hep-th/0103159] [INSPIRE].

[7] H. Aoki, S. Iso, H. Kawai, Y. Kitazawa and T. Tada, Space-time structures from IIB matrix model, Prog. Theor. Phys. 99 (1998) 713 [hep-th/9802085] [InSPIRE].

[8] J. Nishimura, T. Okubo and F. Sugino, Systematic study of the $\mathrm{SO}(10)$ symmetry breaking vacua in the matrix model for type IIB superstrings, JHEP 10 (2011) 135 [arXiv: 1108.1293] [INSPIRE].

[9] S.-W. Kim, J. Nishimura and A. Tsuchiya, Expanding $(3+1)$-dimensional universe from a Lorentzian matrix model for superstring theory in $(9+1)$-dimensions, Phys. Rev. Lett. 108 (2012) 011601 [arXiv:1108.1540] [INSPIRE].

[10] Y. Ito, S.-W. Kim, Y. Koizuka, J. Nishimura and A. Tsuchiya, A renormalization group method for studying the early universe in the Lorentzian IIB matrix model, PTEP 2014 (2014) 083B01 [arXiv:1312.5415] [INSPIRE].

[11] Y. Ito, J. Nishimura and A. Tsuchiya, Power-law expansion of the Universe from the bosonic Lorentzian type IIB matrix model, JHEP 11 (2015) 070 [arXiv: 1506.04795] [INSPIRE].

[12] S.-W. Kim, J. Nishimura and A. Tsuchiya, Expanding universe as a classical solution in the Lorentzian matrix model for nonperturbative superstring theory, Phys. Rev. D 86 (2012) 027901 [arXiv: 1110.4803] [INSPIRE].

[13] S.-W. Kim, J. Nishimura and A. Tsuchiya, Late time behaviors of the expanding universe in the IIB matrix model, JHEP 10 (2012) 147 [arXiv:1208.0711] [INSPIRE].

[14] A. Chaney, L. Lu and A. Stern, Matrix model approach to cosmology, Phys. Rev. D 93 (2016) 064074 [arXiv:1511.06816] [INSPIRE].

[15] A. Chaney, L. Lu and A. Stern, Lorentzian fuzzy spheres, Phys. Rev. D 92 (2015) 064021 [arXiv: 1506.03505] [INSPIRE].

[16] A. Stern, Matrix model cosmology in two space-time dimensions, Phys. Rev. D 90 (2014) 124056 [arXiv: 1409.7833] [INSPIRE].

[17] H.C. Steinacker, Emergent gravity on covariant quantum spaces in the IKKT model, JHEP 12 (2016) 156 [arXiv:1606.00769] [INSPIRE].

[18] H. Steinacker, Emergent geometry and gravity from matrix models: an introduction, Class. Quant. Grav. 27 (2010) 133001 [arXiv:1003.4134] [INSPIRE].

[19] D. Klammer and H. Steinacker, Cosmological solutions of emergent noncommutative gravity, Phys. Rev. Lett. 102 (2009) 221301 [arXiv:0903.0986] [InSPIRE].

[20] H.S. Yang, Emergent spacetime and cosmic inflation I \& II, arXiv:1503.00712 [INSPIRE].

[21] H.S. Yang, Emergent spacetime and the origin of gravity, JHEP 05 (2009) 012 [arXiv:0809.4728] [INSPIRE].

[22] K. Tomita, Fluctuations of the cosmic background radiation appearing in the 10-dimensional cosmological model, PTEP 2015 (2015) 123E01 [arXiv: 1511.08583] [INSPIRE].

[23] A. Chatzistavrakidis, Dynamical phase space from a $\mathrm{SO}(d, d)$ matrix model, Phys. Rev. D 90 (2014) 121502 [arXiv:1407.7054] [INSPIRE]. 
[24] Y. Ito, J. Nishimura and A. Tsuchiya, Large-scale computation of the exponentially expanding universe in a simplified Lorentzian type IIB matrix model, PoS (LATTICE 2015) 243 [arXiv: 1512.01923] [INSPIRE].

[25] K.N. Anagnostopoulos, T. Azuma and J. Nishimura, Monte Carlo studies of the spontaneous rotational symmetry breaking in dimensionally reduced super Yang-Mills models, JHEP 11 (2013) 009 [arXiv: 1306.6135] [INSPIRE]. 